\title{
Biodiversidade Botânica para Formulações de Xampus Naturais ou Orgânicos: um levantamento etnobotânico a partir de patentes
}

\author{
Botanical Biodiversity for Natural or Organic Shampoo Formulations: a \\ patent-based ethnobotanic mapping
}

\author{
Lucas dos Santos Cruz ${ }^{1}$ \\ Vivianni Marques Leite dos Santos ${ }^{1}$ \\ ${ }^{1}$ Universidade Federal do Vale do São Francisco, Petrolina, PE, Brasil
}

\begin{abstract}
Resumo
O setor de cosméticos sustentáveis possui diversas fórmulas com declaração de atenderem ao conceito natural ou orgânico. Diante da grande biodiversidade brasileira, este artigo contém resultados acerca do mapeamento, em nível mundial, de patentes para formulações naturais ou orgânicas de xampus, com vistas a novos desenvolvimentos, tipicamente brasileiros. Para isso, foi realizada busca de anterioridade usando a plataforma Orbit. Destaca-se que a grande maioria (85\%) das 350 famílias de patentes, associadas às formulações de xampus naturais ou orgânicos depositadas de 2000 a 2020, não inclui o nome científico da planta utilizada, impedindo completa identificação, o que pode estar associado a uma decisão do titular para dificultar o uso indevido da invenção. Ademais, as espécies Zingiber spp. e Polygonaceae são as mais utilizadas e, com base no crescente número de patentes, conclui-se pelo alto potencial de uso etnobotânico para novas formulações de xampus.
\end{abstract}

Palavras-chave: Patente. Cosmético. Atóxico. Produto Natural.

\begin{abstract}
The sustainable cosmetics sector has many formulas in compliance with the natural or organic concept. Considering the great Brazilian biodiversity, this study shows results about the worldwide mapping of the patents based in natural or organic formulations of shampoos, aiming new developments typically Brazilian. A search was conducted using the ORBIT platform. It is noteworthy that the majority (85\%) of the 350 patent families associated with formulations of natural or organic shampoos deposited from 2000 to 2020 does not include the scientific name of the plant used. This prevents full identification, which may be associated with a decision by the assigner to hinder misuse of the invention. In addition, the species Zingiber spp. and Polygonaceae are the most used and, based on the growing number of patents, it is concluded that there is a high potential for ethnobotanical use for new shampoo formulations.
\end{abstract}

Keywords: Patent. Cosmetic. Non-toxic. Natural Product.

Área Tecnológica: Propriedade Intelectual. Tecnologia. 


\section{Introdução}

O setor de cosméticos naturais é uma alternativa sustentável que possibilita diversas vantagens, como redução do uso de insumos poluentes, valorização de recursos naturais, assim como da riqueza fitoecológica e promoção da saúde e bem-estar. Caracterizado, principalmente, por transmitir sensação de segurança e de compromisso com a consciência ambiental do público, que, para essa finalidade, utiliza em grande parte ingredientes de composição atóxica e vegetais inalterados por processos químicos (MIGUEL, 2011).

Algumas classificações existem para o que Lyrio et al. (2011) nominaram de biocosméticos quando se referiram aos cosméticos isentos de "conservantes sintéticos, adubos químicos, minerais e artificiais", de modo que, para isso, o produto deve passar por um processo rigoroso de cuidados com os componentes da fórmula, resultando em ações que contribuam para a sustentabilidade ecológica. A proporção desses ingredientes na fórmula é um dos requisitos para diferenciar natural de orgânico.

O mercado para esses produtos possui uma crescente adesão ao longo dos anos, motivada principalmente pela preocupação com o meio ambiente e a alta concentração de nutrientes desses produtos, porém, ainda há uma grande dificuldade por parte dos consumidores em identificar a autenticidade e a integridade desses produtos, bem como para diferenciar as diversas denominações explícitas nos rótulos e que podem confundir os usuários (ALCADE, 2008).

Segundo Magalhães (2018), o Brasil, com sua riqueza ecológica, se destaca como um território favorável para a produção de cosméticos orgânicos, em contrapartida, a comercialização de tais produtos é dificultada por falta de uma legislação sanitária local que dê suporte à fiscalização da autenticidade dos rótulos denominados biocosméticos.

Exposto à vulnerabilidade dos consumidores e à carência de um padrão estabelecido, surgiram organizações de alcance mundial, principalmente empresas como a Natrue e COSMOS-standard, que atuam como certificadoras de cosméticos naturais e orgânicos, possuindo classificações distintas para cada uma delas, ambos são compostos de ingredientes obtidos sem alteração química, de forma física, direta ou por meio de processos que não reproduzam danos ambientais, além disso, para o conceito orgânico, exige-se ainda que a estrutura genética não tenha sido alterada por processos biotecnológicos, possuindo uma linha de produção favorável para a preservação do meio ambiente (BARROS; BARROS, 2020).

Quando é necessário desenvolver cosméticos sustentáveis, as plantas são as fontes mais promissoras, possuindo uma grande quantidade de espécies bem-sucedidas em diversas formulações com benefícios estéticos e medicinais (MIGUEL, 2011). Diversas plantas catalogadas com um alto teor de saponinas se revelam potenciais componentes para produtos higienizantes, como xampus (KREGIEL et al., 2017).

O entendimento de vegetais de interesse para a indústria cosmética já foi utilizado em Wisetkomolmat, Pongsakorn e Sommanof (2019), que ressaltaram a importância de se conduzir estudos mais amplos sobre essas espécies, como composição fitoquímica, uso etnobotânico e dinâmica ecológica, para resultar numa melhor conservação sobre espécies de importância econômica.

Para melhor entendimento da situação sobre uma tecnologia, a prospecção de patentes permite alcançar diversos objetivos sobre informações da proteção da propriedade intelectual, 
como conhecimento do histórico, distribuição geográfica, detentores, autores, tempo de duração legal, bem como seu comportamento no mercado (PARANHOS; RIBEIRO, 2018). Estudos prospectivos no setor cosmético, realizados por Medeiros (2018), revelaram grande uso de tensoativos naturais, moléculas que, em geral, são obtidas a partir de plantas e que possibilitam a formulação de cosméticos higienizantes, como xampus.

As plantas são os principais ingredientes para formulação de cosméticos sustentáveis, devido à sua capacidade de produzir de forma natural e pelo fato de terem diversas moléculas de interesse para fins estéticos e de bem-estar (MIGUEL, 2011). Trabalhos de Wisetkomolmat, Pongsakorn e Sommanof (2019) mostram que promover o conhecimento etnobotânico a partir do uso popular das plantas é uma etapa fundamental para explorar recursos naturais e desenvolver cosméticos de interesse comercial.

Com o intuito de contribuir com a formulação de produtos menos danosos para os consumidores, maior conhecimento sobre as tecnologias já utilizadas e protegidas por meio de patentes envolvendo fórmulas de xampus naturais e orgânicos, bem como possibilitar melhor compreensão acerca da composição botânica, o objetivo deste estudo aborda o mapeamento dos desenvolvimentos tecnológicos protegidos por patentes no mundo, com uso de flora botânica para formulação de xampus naturais e orgânicos.

\section{Metodologia}

Para o levantamento etnobotânico, foram realizadas pesquisas acerca das patentes ao longo dos últimos 20 anos, utilizando a plataforma Orbit intelligence, buscando, no título ou no resumo, as palavras-chave shampoo + AND, natural + AND, organic + AND, plants + , com cada palavra sendo adicionada em diferentes etapas, durante a busca na plataforma de dados, sem restrições quanto ao legal status (depositada, concedida, etc.). Com isso, foi possível visualizar diferenças na classificação cosmética, como dados envolvendo os segmentos da forma orgânica e também natural. Cada patente foi analisada, e foram selecionadas aquelas contendo uso de espécies botânicas, desse modo, a pesquisa foi direcionada ao xampu natural ou orgânico contendo plantas. Para tanto, com base na análise, foram utilizadas as palavras-chave (Shampoo) AND (Plants) AND (Natural OR Organic).

Os resultados da pesquisa foram obtidos a partir da plataforma nos meses de julho até agosto de 2020, cujos dados foram tratados por meio de planilhas eletrônicas para geração de gráficos, proporcionando melhor visualização do cenário de patentes.

As espécies que foram possíveis de serem identificadas pelo nome científico correto foram registradas diretamente num quadro, já aquelas que foram descritas pelo nome popular, a descrição foi feita até o gênero da planta, indicado por meio de artigos científicos, sobretudo estudos etnobotânicos, encontrados pelo Google Scholar como base de dados.

Desse modo, o registro, neste texto, seguiu a classificação taxonômica botânica, os níveis de família, gênero e espécie, correspondentes às plantas utilizadas em patentes de xampus naturais e orgânicos, cada exemplar foi registrado em quadro para obtenção de mais dados sobre o vegetal de interesse, como o uso correto da descrição taxonômica e a quantidade de vezes mencionada. 


\section{Resultados e Discussão}

Os resultados que melhor atenderam à especificidade de busca com relação aos cosméticos foram obtidos utilizando a combinação de diferentes palavras-chave associados à palavra "SHAMPOO+", conforme etapas a seguir: acrescentada a palavra natural (AND NATURAL), a busca gerou 1.444 resultados. Na etapa seguinte, substituiu-se o último termo por (AND ORGANIC), sendo obtidos 750 resultados. A ideia foi obter informações separadas para natural e orgânico. Na terceira etapa, utilizou-se os termos da etapa 1 e 2 combinados, rendendo 110 achados. Esse número foi reduzido quando foi adicionado o termo +PLANTS, restringindo a busca por tecnologias que utilizam plantas, sendo obtidos apenas 25 famílias de patentes (Tabela 1).

Desse modo, verifica-se que xampus que seguem a tendência de cosmético verde são majoritariamente registrados como produtos naturais do que orgânicos. Supõe-se que os desenvolvimentos de produtos naturais em maior número se devem à menor quantidade de critérios exigidos para uso desse termo, os quais foram detalhadamente descritos por Barros $e$ Barros (2020).

Tabela 1 - Resultado das buscas por palavras-chave

\begin{tabular}{ccc} 
Etapa & Palavras-chave & Resultados \\
1 & SHAMPOO? AND NATURAL & 1.444 \\
2 & SHAMPOO? AND ORGANIC & 750 \\
3 & SHAMPOO? AND ORGANIC AND NATURAL & 110 \\
4 & SHAMPOO? AND ORGANIC AND & 25 \\
5 & NATURAL+ AND PLANT? & 430 \\
\hline
\end{tabular}

Fonte: Elaborada pelos autores deste artigo

Após a última etapa do levantamento (etapa 5 - Tabela 1), que resultou em 430 famílias de patentes, a análise dos resumos e das reivindicações permitiu identificar 350 desenvolvimentos majoritariamente naturais ou orgânicos. Para estas, conforme apontam Paranhos e Ribeiro (2018), foram analisados a composição, o processo de produção, os responsáveis inventores e titulares e a(s) reinvindicação(ões) sobre o processo e/ou produto inovador(es).

Com relação à proteção dos direitos comerciais de uma fórmula cosmética, o legal status é importante, pois revela a situação da patente com relação ao andamento do processo de pedido de proteção, e, indiretamente, aqueles dados subsidiam conclusões sobre o gerenciamento das patentes, ou seja, se os titulares estão monitorando adequadamente todas as etapas, como atendimento de exigências e outros tipos de notificações ou mesmo cumprimento dos prazos de pagamentos de taxas.

Verificou-se que não há patente expirada (Figura 1), ou seja, patentes concedidas que atingiram o limite temporal de proteção. Isso revela que as patentes concedidas ainda estão aptas para seus licenciamentos ou transferências, consideradas as reivindicações a partir dos seus titulares. Em outras palavras, nenhuma das patentes concedidas atingiu seu período máximo de proteção. 
Há elevado número de patentes para as quais não foram pagas as taxas (163 patentes ou $46,6 \%$ ), de modo que elas têm status lapsed, ou seja, não foram adequadamente mantidas ou foram abandonadas pelos seus titulares, os quais devem se responsabilizar pela manutenção de suas patentes. Ademais, somadas as patentes revogadas, verifica-se que, em sua maioria (203 patentes ou 58,0\%), os depósitos de patente estão inativos, ou seja, perderam efeito legal de proteção. Acrescenta-se que, nos casos em que uma patente se tornou inativa, mas já foi publicada, seu conteúdo passou para domínio público, podendo ser utilizado por quaisquer terceiros sem necessidade de autorização a partir do titular.

Por outro lado, existem 106 patentes ativas com status pendente (30,3\%), ou seja, o pedido não foi abandonado, mas também ainda não foi examinado e, finalmente, outras 41 patentes foram concedidas $(11,7 \%)$ e, portanto, estão aptas para sua aplicação efetiva no mercado. Nesse sentido, há 147 potenciais produtos ou processos com uso da biodiversidade botânica em formulações de xampus.

Figura 1 - Número de patentes por status legal

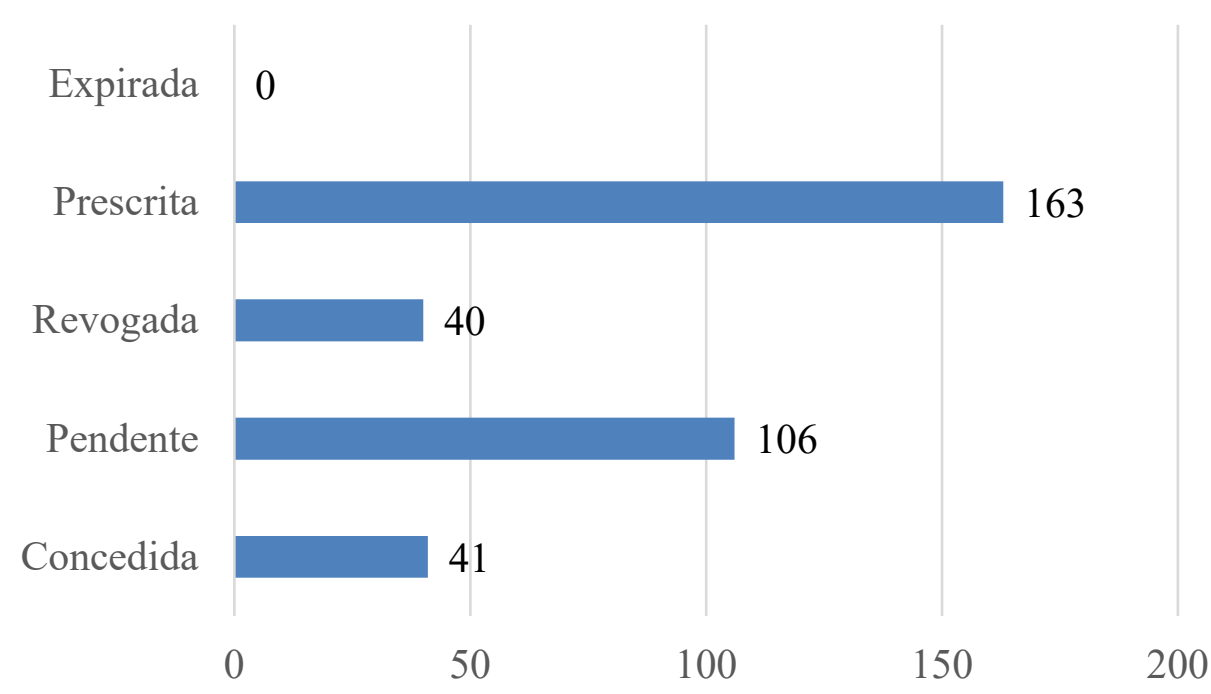

Fonte: Elaborada pelos autores deste artigo

Com base na análise da quantidade de patentes ao longo dos anos (Figura 2), verifica-se um elevado número em 2001, seguido por nove anos com número pequeno quando comparado ao ano de 2001. Por outro lado, em 2011, houve aumento e comportamento crescente desde então. Os números de patentes nos anos de 2019 e 2020 não são conclusivos, já que existem os períodos de sigilo, durante os quais as patentes não são publicadas, logo aqueles números descritos na Figura 2 devem ser significativamente menores. Isso posto, estima-se que o desenvolvimento na área continua em ascensão. 
Figura 2 - Número de depósito de patentes nos últimos 20 anos

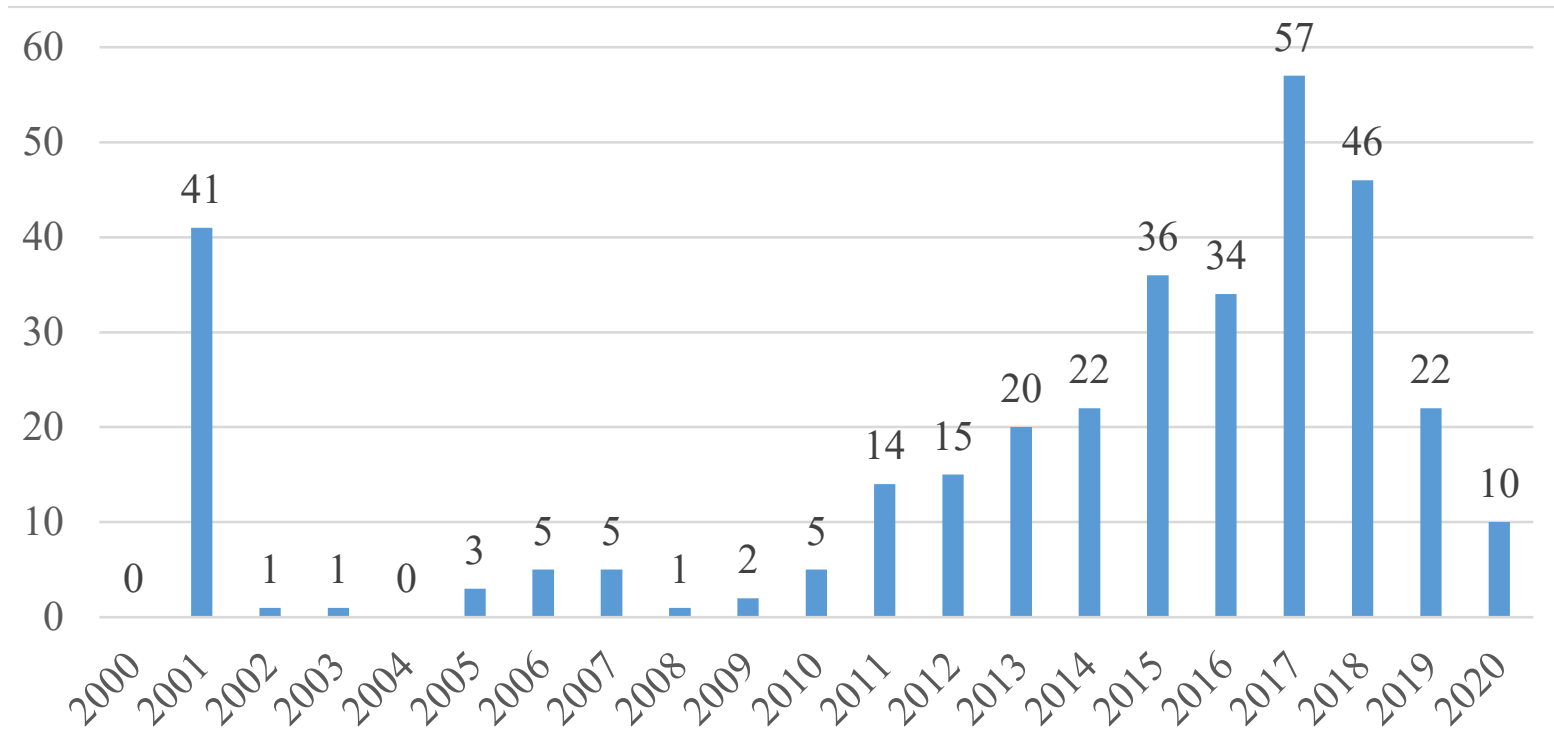

Fonte: Elaborada pelos autores deste artigo a partir dos dados obtidos usando o Orbit (2020)

Importante destacar que a maioria das patentes depositadas em 2001 corresponde a desenvolvimentos a partir de um mesmo titular e autor Chinês (Chen Huinian), com 58,5\% (24) do total naquele ano, no mundo. Outra curiosidade é que o referido autor atribuiu 19 títulos idênticos para patentes que solicitou proteção para diferentes formulações com espécies vegetais como aditivos e outras cinco para formulações contendo espécies vegetais com foco na proteção capilar. Ademais, as diferentes espécies são, majoritariamente, nativas da Ásia. Acrescenta-se que o titular e autor não é responsável pelos novos desenvolvimentos desde então.

A riqueza botânica, aliada à sua devida exploração por pesquisadores de países como a China, Coreia e Japão, vem revelando resultados promissores há anos. Em 2012, os estudos etnobotânicos, incluindo a medicinal tradicional chinesa, apontaram uma série de compostos ou moléculas de grande interesse para a medicina e a cosmetologia (WIART, 2012), e é justamente nestes países que se concentram as maiores quantidades de patentes, conforme Figura 3.

Figura 3 - Quantidade de Famílias de patentes por país de primeira prioridade

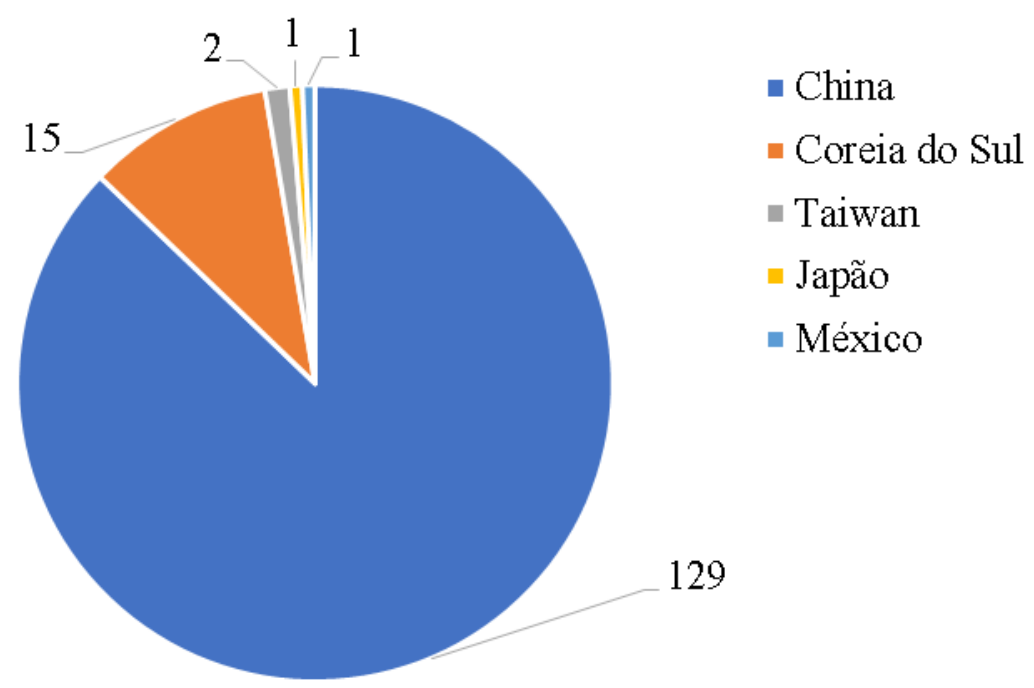

Fonte: Elaborada pelos autores deste artigo 
Durante o estudo, nem todas as plantas puderam ser identificadas, essa dificuldade se deu pela falta de utilização do nome científico. Assim, foram identificadas 62 plantas, as quais estão registradas na Tabela 2. Mesmo entre estas, alguns achados se limitaram até o nível taxonômico da família, como é o caso da Polygonaceae. Observa-se ainda que há uma linha a mais na Tabela que se deve a uma mesma planta, a Zanthoxylum, que foi adequadamente descrita em uma patente (Zanthoxylum americanum) e não descrita em outras três patentes (Zanthoxylum spp.)

Entre as demais patentes com espécies descritas corretamente, destaca-se aquela em que foi usada a Ginko biloba, informando claramente o material vegetal utilizado. Por outro lado, algumas contêm apenas a descrição do gênero, como ocorreu em seis patentes que incluem o uso do Aloe, mas não deixam claro se, por exemplo, foi utilizada Aloe arborescens, espécie adequadamente descrita em dois documentos de patente, ou ainda se foi utilizada a Aloe vera, que obteve correta descrição em dois outros documentos.

Tabela 2 - Espécies vegetais em patentes

EsPÉCIE CITADA

NÚMERO DE VEZES MENCIONADA
CORRETA DESCRIÇÃo TAXONÔMICA

(Ao Menos UMA Vez)

$$
\begin{gathered}
\text { Acorus gramineus } \\
\text { Agave lechuguilla } \\
\text { Ageratum spp. } \\
\text { Allium sp. } \\
\text { Aloe arborescens } \\
\text { Aloe spp. } \\
\text { Aloe vera } \\
\text { Anethum graveolens } \\
\text { Angelica spp. } \\
\text { Artemisia spp. } \\
\text { Bupleurum spp. } \\
\text { Camellia spp. } \\
\text { Canavalia gladiata } \\
\text { Carica papaya } \\
\text { Carica sp. }
\end{gathered}
$$

Cerasus sp.

Chamaecyparis $s p$.

Cinnamomum spp.

Chlorogalum pomeridianum

Citrus spp.

Cyperus rotundus

Dahuria angelica

Eclipta spp.

Ganoderma lucidum (fungo)

Gastrodia elata

Ginko biloba
1

1

3

1

2

6

2

1

8

2

3

4

1

1

1

1

1

2

1

6

1

1

4

2

1

1
Não

Não

Não

Não

Não

Não

Não

Não

Não

Não

Sim

Não

Não

Não

Não

Não

Não

Não

Não

Não

Não

Sim

Não

Sim

Não

Sim 


\section{ESPÉCIE CITADA}

NÚMERO DE VEZES MENCIONADA
CORRETA DESCRIÇÃo TAXONÔMICA

(Ao Menos Uma Vez)

\section{Gleditsia sinensis}

Gleditsia sp.

Heena sp.

Helianthus annuus

Hibiscus spp.

Honeylocust spp.

Humulus japonicus

Humulus lupulus

Jatropha cinérea

Jatropha cuneata

Jatropha dioica

Ligusticum chuanxiong

Ligustrum lucidum

Lonicera japônica

Magnolia sp.

Matricaria spp.

Mentha piperita

Olea spp.

Paeonia spp.

Panax spp.

Persea americana

Polygonaceae (família)

Purslanes sp.

Quillaja saponária

Rabdosia sp.

Rhynchosia sp

Rumex crispus

Sapindus mukurossi

Sesame sp.

Solanum nigrum

Sophora angustifolia

Stephania cepharantha

Thymus sp.

Vitis spp.

Zanthoxylum americanum

Zanthoxylum spp.

Zingiber spp.

2

1

1

1

3

1

1

1

1

1

1

1

1

1

1

5

1

3

3

7

1

15

1

4

1

1

1

1

1

1

1

1

1

2

1

3
Não

Não

Não

Não

Não

Não

Não

Não

Sim

Sim

Sim

Não

Sim

Não

Não

Não

Não

Não

Não

Não

Não

Não

Não

Não

Não

Não

Não

Não

Não

Não

Sim

Não

Não

Não

Sim

Não

Não

Fonte: Elaborada pelos autores deste artigo 
Nesse sentido, mesmo entre as 62 plantas identificadas, com aplicação em desenvolvimentos tecnológicos para formulações de xampus, com pedidos de proteção por patente, 53 plantas não estão adequadamente descritas. Entende-se por inadequadamente descritas aquelas que não obedecem às regras de nomenclatura da taxonomia botânica, isso pode ocorrer de diversas formas, como o não emprego da nomenclatura binomial (composta de gênero e epiteto específico). Desse modo, quando uma patente menciona apenas o gênero da planta utilizada, não deixa claro qual espécie está sendo utilizada entre diversas outras que podem existir no mesmo nível de organização taxonômica. Adiciona-se que, nas regras taxonômicas, sp. refere-se à substituição de um indeterminado epiteto específico quando se tem a necessidade de descrever uma espécie específica em um gênero e spp. refere-se à possibilidade de múltiplas espécies dentro de um mesmo gênero (RAVEN; EICHHORN; EVERT, 2014).

Espécies do mesmo gênero podem se diferenciar bastante em composição fitoquímica e distribuição geográfica, o que pode alterar significativamente propriedades importantes em uma reinvindicação de patente. A exemplo do gênero Ziziphus, que possui a espécie Ziziphus jujuba originada na África e Austrália, que possui uma ampla utilização medicinal em países da Ásia, além de sua aplicação em formulações cosméticas, tal como na produção de xampus na Ásia Ocidental, no Omã e regiões próximas desse país (HOSSAIN, 2019). De acordo com Elaloui et al. (2016), a grande quantidade de ácidos graxos livres, como ácido linolênico e ácido palmítico, resulta na sua promissora aplicação em formulações de cosméticos. Em contrapartida, outra espécie do Ziziphus joazeiro, aquela nativa da região Nordeste do Brasil, também tem aplicação na indústria cosmética, porém isso ocorre devido a outros compostos e suas concentrações, tal como as saponinas, que são moléculas tensoativas com grande poder de espuma (DO NASCIMENTO et al., 2020).

Além da importância em se determinar exatamente o nome de uma espécie, recomenda-se identificar seus nomes tradicionais. Isso é relevante porque quando não aplicado o nome científi$\mathrm{co}$, as espécies costumam ser referidas pelo nome popular, principalmente quando utilizadas com base no conhecimento tradicional associado, aquele passado de gerações para gerações por meio de saberes empíricos. Nesse contexto, destaca-se a contribuição de resultados a partir de estudos etnobotânicos, com registros de plantas com relação a diversos aspectos, como interesse de consumo, identificação científica e nomeações populares. De acordo com Haeruddin, Hairah e Budiman (2017), tais estudos correlacionam de forma bidirecional o saber científico e o tradicional.

Ainda nesse contexto, conclui-se que, quanto aos documentos de patentes, deve-se priorizar o nome científico, uma vez que de fato isso permite identificação completa, ou seja, confere a especificidade de um vegetal como organismo, diante da diversidade de nomes que acompanham as espécies e suas modificações por culturas em todo o mundo. A despeito disso, em apenas $16,1 \%$ dos documentos de patentes, as plantas a partir das quais foram obtidas as formulações para novos xampus foram completamente descritas, ou seja, os inventores utilizaram a nomenclatura científica, de modo que a grande maioria não contém a descrição adequada (Figura 4). Em suma, a não citação adequada das espécies constitui desafio para estudos prospectivos e até mesmo sua reprodução por um técnico na área, de modo que pode até mesmo dificultar a concessão de patente, considerados os requisitos descritos pelo Instituto Nacional da Propriedade Industrial. 
Figura 4 - Correta (sim) ou incorreta (não) descrição taxonômica de plantas em documentos de patentes

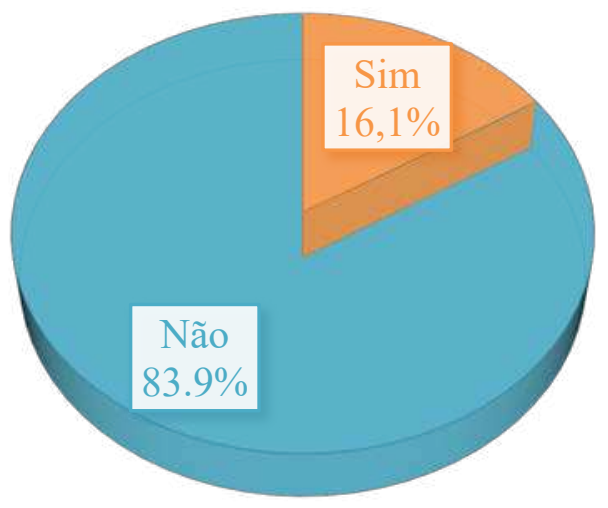

Fonte: Elaborada pelos autores deste artigo

Entre as plantas mais utilizadas nas formulações de xampus (Figura 5), destaca-se a Zingiber spp., mencionada em 18 famílias de patentes diferentes, embora em nenhuma delas houve sequer uma menção pelo nome científico, descritas apenas pelo nome popular ginger, referindo-se ao gengibre. O uso dessa mesma planta em xampus já foi mencionado por Yan, Liu e Qin (2005), quando eles revelaram que, em concentrações adequadas, o extrato do vegetal pode promover benefícios ao cabelo, como combate à perda de cabelo e ação anticaspa. Um estudo de Chavan et al. (2018) explorou a composição do gengibre, destacando moléculas de interesse cosmético e farmacêutico, como sesquiterpenos monocíclicos e compostos polifenólicos. Acrescenta-se que a Figura 4 apresenta somente as plantas que foram utilizadas em duas ou mais patentes, de modo que as 49 outras plantas foram citadas em apenas uma patente.

Figura 5 - Número de espécies mais encontradas

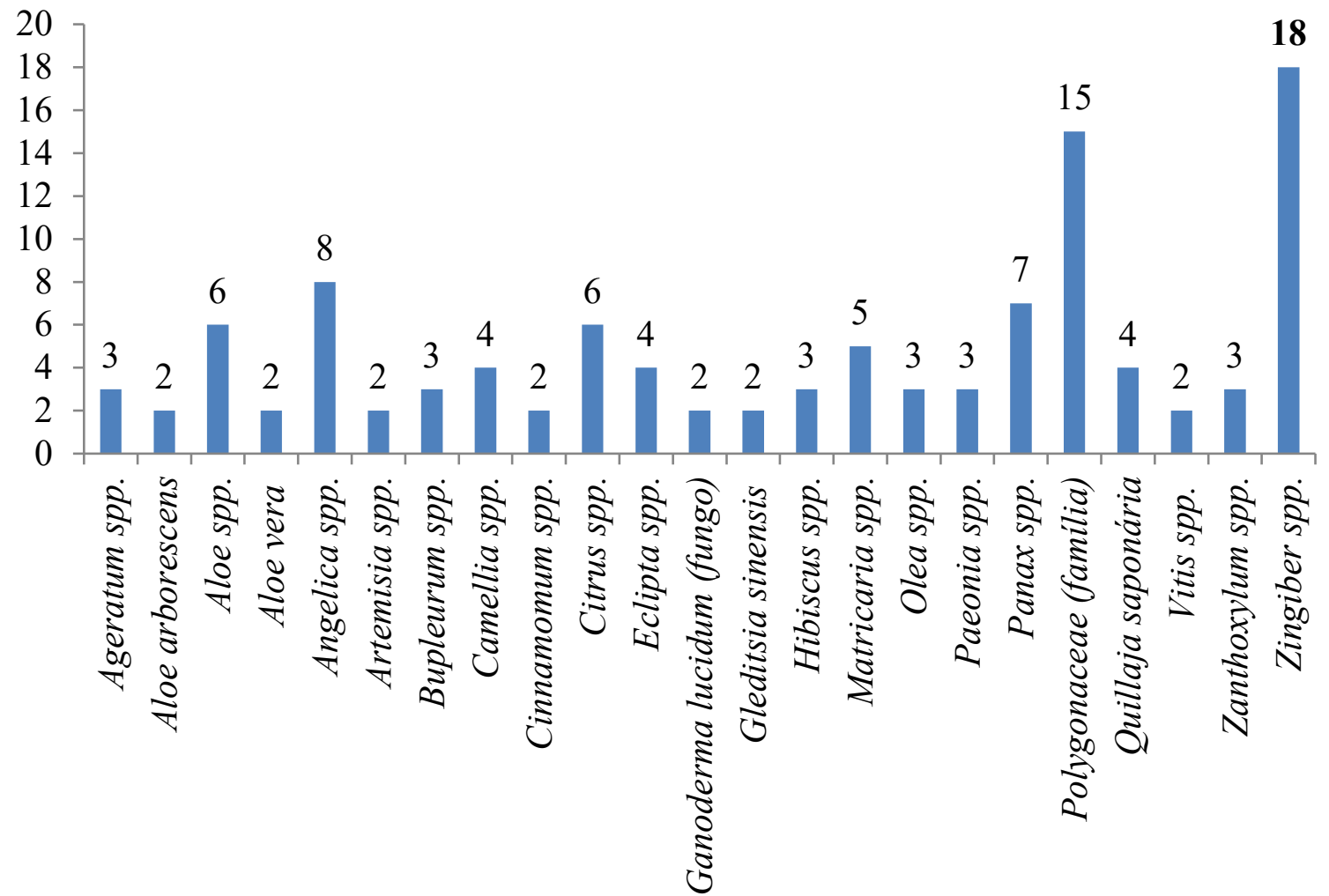

Fonte: Elaborada pelos autores deste artigo 
Adicionalmente, houve referência a outras plantas por nomes não científicos, tal como o termo "chinese medicine extracts", referindo-se a múltiplas espécies vegetais aplicadas na etnobotânica tradicional chinesa, de modo que não foi possível identificar nenhum nível taxonômico para essas plantas ou para os extratos mencionados, com ressalva de que a maioria está descrita nos documentos de patente depositados em 2001 pelo Chinês Chen Huinian.

Curioso mencionar que, além das plantas, uma espécie de fungo foi utilizada, a Ganoderma lucidum, a qual, segundo Taofiq et al. (2017), possui resultados promissores para obtenção de formulações cosmecêuticas, isto é, cosméticos administrados por via oral, ou suplementos consumidos com a finalidade de promover benefícios estéticos e até mesmo fisiológicos, por ser rico em nutrientes como, terpenoides e compostos fenólicos.

O Brasil, apesar da grande biodiversidade, de acordo com os resultados, ainda não dispõe de pedidos de patentes publicados referentes à proteção de formulações de xampus naturais ou orgânicos, embora a Lei n. 9.279, de 14 de maio de 1996 (BRASIL, 1996), não proíba tais procedimentos. Supõe-se que tal fato pode resultar da cultura associada ao não reconhecimento da importância estratégica do desenvolvimento de pesquisas passíveis de proteção para inovação nas mais diversas áreas. Nesse cenário, Leal e Figueiredo (2021) destacam que o País vem sofrendo elevadas perdas no mercado econômico global, devido ao atraso tecnológico que ainda se encontra quando comparado aos demais países.

Por outro lado, apesar de a busca a partir de bases não patentárias não constituir o foco deste estudo, destaca-se que a razão entre o número de famílias de patentes e aquele de publicações científicas identificadas a partir das mesmas palavras-chave utilizando o Orbit é de apenas 0,012, ou seja, muito baixa, apontando que as pesquisas acerca de novas formulações de xampus naturais ou orgânicos estão em sua maioria em níveis de maturidade tecnológica ou Technology Readiness Level (HONGWEI et al., 2021) baixos (resultados de pesquisas publicadas em literatura não patentária), não atendendo ainda aos requisitos de patenteabilidade (INPI, 2021) para uma nova formulação.

Supõe-se que o crescimento de pedidos de depósito de patentes sobre cosméticos naturais e orgânicos acompanha a maior consciência quanto à saúde, à necessidade de redução dos impactos ambientais e ao uso racional da biodiversidade como nicho para desenvolvimento econômico. Ademais, de acordo com Miguel (2011), essa crescente expansão pode estar associada à necessidade de produzir seus próprios produtos, como ocorre com a Yves Rocher, que propõe novos produtos, cumprindo as exigências regulamentais de produção cosmética ou, por outro lado, optam pelo investimento na compra de outras empresas de menor porte, como a LOreal, buscando expandir para novas regiões e atingir consumidores habituados com diferentes tipos de produto de consumo. $\mathrm{O}$ autor acrescenta que, no Brasil, a empresa de maior destaque é a Natura, com diversos certificados nacionais e internacionais, tal como o IBD, ECOCERT, Imaflora, SAN, FSC. A despeito disso, os autores deste artigo, conforme metodologia descrita, não identificaram nenhuma patente com titularidade da referida empresa.

\section{Considerações Finais}

A quantidade de pedidos de proteção envolvendo xampus naturais ou orgânicos com primeira prioridade na China destaca-se no cenário mundial, a partir do qual estima-se um grande 
aproveitamento do conhecimento etnobotânico local e do conhecimento tradicional associado. Por outro lado, com base na análise do número total de patentes por ano, esse setor tende a crescer cada vez mais, sendo verificado significativo avanço no ano de 2011, com manutenção do comportamento crescente, chegando a um recorde em 2017. Assim, a perspectiva é de que nos próximos anos seja mantida a alta frequência de proteção de desenvolvimentos tecnológicos por meio de patentes.

A despeito do crescimento no pedido de depósitos, observa-se um grande número de patentes inativas, em que $163(46,6 \%)$ encontram-se prescritas por falta de pagamento de taxas e $40(11,4 \%)$ foram revogadas, permitindo observar que ainda há baixa frequência de sucesso entre tais patentes. Identificação esta que merece atenção em estudos futuros.

Neste estudo, todas as patentes encontradas com as palavras-chave que remetem a xampus naturais ou orgânicos usavam pelo menos uma planta, tal que as drogas vegetais ou partes específicas e extraídas da planta constituem ingredientes relevantes para tais formulações cosméticas de aplicação capilar. Porém, cabe destacar que tais depósitos precisam de padronização, pois a maior parte utilizou uma descrição inadequada ou incompleta, correspondendo a $85 \%$ das famílias de patentes. Isso pode ser um obstáculo para a decisão sobre sua utilização, dada a impossibilidade de identificação da planta, ou seja, dos insumos para o processo de produção. Ademais pode ser decisão dos titulares para dificultar uso das formulações sem a devida autorização.

Finalmente, com base nos resultados, conclui-se que as espécies Zingiber spp. e Polygonaceae (família) são as mais utilizadas no período, sendo citadas, respectivamente, em 18 e 15 famílias de patentes, e que o mercado de xampus naturais ou orgânicos tende a sofrer grande influência do uso etnobotânico das plantas, embora com desafios acerca da correta descrição das espécies, fazendo-se necessária a adoção de nomenclatura científica, como forma de definir exatamente os ingredientes vegetais das formulações.

\section{Perspectivas Futuras}

Com base nas espécies vegetais identificadas, novos estudos podem ser realizados acerca dos seus usos etnobotânicos para formulação de outros tipos de cosméticos ou ainda estudos para identificação da ocorrência natural das referidas espécies, incluindo as condições climáticas e de solo daquelas localidades.

Também se sugerem estudos acerca da análise da correlação entre a biodiversidade botânica dos países e o número de pedidos de patentes envolvendo utilização de espécies vegetais, com primeira prioridade naqueles países, além de estudos sobre as causas do elevado número de patentes inativas ao longo dos anos.

\section{Referências}

ALCADE, Teresa. Cosmética natural y ecológica. Offarm, [s.l.], v. 27, n. 9, p. 96-102, 2008.

BARROS, Cleber; BARROS Rosana Bevenuto Guilhen. Natural and Organic Cosmetics: Definition and Concepts. Preprints, [s.l.], 2020. 
BRASIL. Lei n. 9.279, de 14 de maio de 1996. Disponível em: http://www.planalto.gov.br/ ccivil_03/leis/19279.htm. Acesso em: 30 jul. 2021.

CHAVAN, Jaykumar J. et al. Micropropagation, metabolite profiling, antioxidant activities and chromatographic determination of bioactive molecules across in vitro conditions and subsequent field cultivation stages of Shampoo Ginger (Zingiber zerumbet L. Roscoe ex Sm). Biocatalysis and Agricultural Biotechnology, [s.l.], v. 16, p. 78-89, 2018.

COSMOS. Cosmetics Organic and Natural Standard. Versão 3.1. 2020. Disponível em: https:// www.cosmos-standard.org/about-the-cosmos-standard. Acesso em: 27 jul. 2020.

DO NASCIMENTO, Alan M. et al. Ziziphus joazeir o, a Saponin-Rich Brazilian Medicinal Plant: Pharmacognostic Characterization of Bark and Leaves. Revista Brasileira de Farmacognosia, [s.l.], p. 1-9. 2020.

ELALOUI, Meriem et al. Phytoconstituents of leaf extracts of Ziziphus jujuba Mill. plants harvested in Tunisia. Industrial Crops and Products, [s.l.], v. 83, p. 133-139, 2016.

GARCÍA, Díaz et al. Elaboración de gel y shampoo para el control de las manifestaciones clínicas de la caspa (Dermatitis Seborreica) elaborado a partir de extracto de jengibre (Zingiber officinale). Estudio Piloto, [s.l.], p. 59-59, 2017.

HONGWEI, Li et al. A Conceptual Technology Readiness Loop Model Through Systematic Review and Thematic synthesis. Turkish Journal of Computer and Mathematics Education, [s.l.], v. 12, n. 11, 2021.

HOSSAIN, Mohammad A. A phytopharmacological review on the Omani medicinal plant: Ziziphus jujube. Journal of King Saud University-Science, [s.l.], v. 4, n. 31, p. 1.352-1.357, 2019.

INPI - INSTITUTO NACIONAL DA PROPRIEDADE INDUSTRIAL. Manual Básico para Proteção por Patentes de Invenções, Modelos de Utilidade e Certificados de Adição. 2021. Disponível em: https://www.gov.br/inpi/pt-br/servicos/patentes/guia-basico/ManualdePatentes20210706.pdf. Acesso em: 30 jul. 2021.

HAERUDDIN, Herni Johan; HAIRAH, Ummul; BUDIMAN, Edy. Ethnobotany database: Exploring diversity medicinal plants of Dayak tribe Borneo. In: 2017 4TH INTERNATIONAL CONFERENCE ON ELECTRICAL ENGINEERING, COMPUTER SCIENCE AND INFORMATICS (EECSI), p. 1-6, 2017. Anais. [S.l.], 2017.

KREGIEL, Dorota et al. Saponin-based, biological-active surfactants from plants. Application and Characterization of Surfactants, [s.l.], p. 183-205, 2017.

LEAL, Carlos Ivan Simonsen; FIGUEIREDO, Paulo Negreiros de. Inovação tecnológica no Brasil: desafios e insumos para políticas públicas. Revista de Administração Pública, [s.l.], v. 55, n. 3, 2021.

LYRIO, Eyna S. et al. Recursos vegetais em biocosméticos: conceito inovador de beleza, saúde e sustentabilidade. Natureza On Line, [s.l.], v. 9, n. 1, p. 47-55, 2011.

MAGALHÃES, Lorena Severiano de. Cosméticos orgânicos: uma tendência crescente no mercado ainda pouco conhecida. 2018. 26p. Monografia (Bacharelado) - Universidade Federal de Ouro Preto, Minas Gerais, 2018. 
MEDEIROS, Dandara Martins Costa. Prospecção tecnológica no setor de tensoativos na indústria de cosméticos. 2018. 69p. Trabalho de Conclusão de Curso (Graduação em Química com Contribuições Tecnológicas) - Universidade Federal do Rio de Janeiro, Rio de Janeiro, 2018.

MIGUEL, Laís Mourão. Tendências do uso de produtos naturais nas indústrias de cosméticos da França. Revista Geográfica de América Central, [s.l.], v. 2, p. 1-15, 2011.

NATRUE. Label: requirements to be met by natural and organic cosmetics. Versão 3.8. 2019. Disponível em: https://www.natrue.org/natrues-standard-update-version-3-8-2/. Acesso em: 27 jul. 2020.

PARANHOS, Rita de Cássia Santos; RIBEIRO, Núbia Moura. Importância da Prospecção Tecnológica em base em patentes e seus objetivos da busca. Cadernos de Prospecção, Salvador, v. 11, n. 5 , p. $1.274-1.292,2018$.

RAVEN, Peter H.; EICHHORN, S. E.; EVERT, R. F. Biologia Vegetal. 8. ed. Rio de Janeiro: Guanabara Koogan, 2014.

TAOFIQ Oludemi et al. The potential of Ganoderma lucidum extracts as bioactive ingredients in topical formulations, beyond its nutritional benefits. Food and Chemical Toxicology, [s.l.], v. 108, p. 139-147, 2017.

WIART, Christophe. Medicinal plants of China, Korea, and Japan: bioresources for tomorrow's drugs and cosmetics. CRC Press, [s.l.], 2012.

WISETKOMOLMAT, Jiratchaya; PONGSAKORN, Suppakittpaisarn; SOMMANO, Sarana Rose. Detergent Plants of Northern Thailand: Potential Sources of Natural Saponins. Resources 8, [s.l.], v. 10, n. 1, 2019.

YAN, Zi-jun; LIU Yong; QIN Chao-guo. Application of ginger in shampoo [J]. Shaanxi Chemical Industry, [s.l.], v. 10, 2005.

\section{Sobre os Autores}

\section{Lucas dos Santos Cruz}

E-mail: lucascruz790@gmail.com

ORCID: http://orcid.org/0000-0001-7213-7039

Graduando de Farmácia.

Endereço profissional:Universidade Federal do Vale do São Francisco, Campus Sede, Av. José de Sá Maniçoba, s/n, Centro, Petrolina, PE. CEP: 56304-917.

\section{Vivianni Marques Leite dos Santos}

E-mail: vivianni.santos@gmail.com

ORCID: http://orcid.org/0000-0001-8741-8888

DSc Química.

Endereço profissional: Doutorado em Agroecologia e Desenvolvimento Territorial (PPGADT), Universidade Federal do Vale do São Francisco, Campus Juazeiro, Avenida Antonio Carlos Magalhães, n. 510, Santo Antônio, Juazeiro, BA. CEP: 48902-300. 